\title{
Permanent Magnet Reduction by Current Harmonics Injection for Surface Permanent Magnet Machines
}

\author{
$1^{\text {st }}$ Daniele De Gaetano \\ Power Electronics, Machines and Control Group \\ University of Nottingham \\ Nottingham, UK \\ ORCID: 0000-0002-9770-0171 \\ $3^{\text {rd }}$ Michele Degano \\ Power Electronics, Machines and Control Group \\ University of Nottingham \\ Nottingham, UK \\ ORCID: 0000-0002-9689-1172
}

\author{
$2^{\text {nd }}$ Giacomo Sala \\ Department of Electrical, Electronic and \\ Information Engineering University of Bologna \\ Bologna, IT \\ ORCID: 0000-0002-6374-7713 \\ $4^{\text {th }}$ Chris Gerada \\ Power Electronics, Machines and Control Group \\ University of Nottingham \\ Nottingham, UK \\ ORCID: 0000-0003-4707-4480
}

\begin{abstract}
This paper presents the analysis of multi-harmonic current injection in multi-three phase surface permanent magnet (SPM) machines. An analytical model, extended to a generic harmonic order, is used to describe and determine the no load flux density, the back electromotive force (BEMF) and the electromagnetic torque produced. These are analysed as a function of the permanent magnet span. Based on the BEMF, the harmonics injection technique is adopted to increase the torque in the machine, while keeping the RMS current constant, thus without affecting the Joule losses. In order to understand how the torque is affected by the harmonics injection, considering different PMs rotor span, a comparison with and without injection is presented. It is shown how, thanks to specific harmonics injection, it is possible to reduce the permanent magnet content in SPM machines while generating the same torque performance of a motor with higher PM content, without harmonic injection. The analytical results are finally validated by means of Finite Element Analysis (FEA) considering two different PM rotor span, as a case study, highlighting the benefits of the proposed method.
\end{abstract}

Index Terms-Analytical models, Electric machines, Harmonic injection, Magnetic flux density, Multiphase machines, Permanent magnet machines, Rotating machines.

\section{INTRODUCTION}

The surface permanent magnet (SPM) machines are commonly used in the applications where high torque per volume is required such as aerospace, automotive and transport applications [1], [2]. For high power/torque performance, it is well known that the use of rare earth materials, such as $\mathrm{NdFeB}$ and $\mathrm{SmCo}$, are considered the state of the art, thanks to their high magnetic coercitivity and excellent temperature behaviour. However, because of their high cost in many applications it is mandatory to reduce their use. In literature, many authors presented possible alternatives to the rare earth, including different permanent (PM) machines topologies [3], [4]. In [5], a possible solution to replace the rare earth with ferrite for an interior PM machine is evaluated. A comparison between rare earth and rare earth-free solutions is investigated in [6]. An hybrid PM material is designed and analysed in [7]. Another method to increase the electrical machine torque capability is by using a multi-phase winding design [8]. A technique to rewind classical three-phase stator frames with a generic prime phase order is illustrated in [9]. A novel 9-phase permanent magnet synchronous motor is presented in [10], where is shown how the power/speed range can be extended. Tests and analyses on a dual-three phase permanent magnet motor are reported in [11], showing operation under fault conditions. Other works propose the harmonic injection technique to increase the torque capability. In [13], this is shown for a dual three-phase machine by third harmonic injection technique for both at the same peak and RMS current. This method is also applied to a five-phase unequal tooth SPM machine in [14]. [15] shows the torque capability enhancement of a dual three-phase SPM machine injecting the fifth and seventh current harmonics. The third current harmonic injection is investigated also for a nine-phase machine with high nonsinusoidal BEMF in [16]. Genetic algorithms are implemented in [18] in order to maximize the torque per ampere and optimize the iron utilisation by an optimum flux distribution by harmonic injection.

This work is analysing in detail the effects of the permanent magnet span, of SPM machines, on the spatial harmonics distribution. This is then used to understand how it is possible to reduce the PM content in the machine, without affecting torque performance, through the current harmonic injection technique. The proposed method permits to maintain the RMS current constant with respect to the current control with the only fundamental component, thus the Joule losses are not affected by harmonics injection.

A similar approach has been used in [18], where a ninephase SPM machine is studied via FE for different PM span to increase the torque performance by harmonic injection. However, the investigation is based on the injection until the seventh harmonic. 
The paper is organised as follows. Sec. II shows the flux density at the air-gap and back electromotive force (BEMF) as a function of the PM percentage on the rotor in a 3x3phase SPM machine for different spatial harmonics. Then, the equations for deriving the current amplitudes of each temporal harmonics to inject, to increase the torque for the same RMS current, are presented. In the same section, the torque comparison between the machine with and without harmonics injection is carried out as a function of the PM percentage. The analytical model results are validated via finite element (FE) simulations in Sec. III. The comparison in terms of phase voltage, phase current and torque is carried out for two 3x3 SPM machines with different PM content. The outcomes of the paper are showing how it is possible, thanks to the proposed harmonics injections, modulate the percentage of permanent magnet volume.

\section{AnAlyticAl MODEL}

A simple analytical derivation is here derived for the evaluation of the machine flux density, BEMF and torque. A set of equations is also included to define the current amplitudes to maximise the torque for the same RMS value. The model works under the assumptions of linear materials and radial flux density at the airgap.

\section{A. Flux Density and Back Electro-Motive Force}

An electrical machine generates the torque through the interaction between rotor and stator magnetic field. For a PM machine, the rotor magnetic field contribution is due to the permanent magnets. The magnetic field at the air-gap generated by the rotor, in an SPM machine, for $\rho^{t h}$ spatial harmonics, can be written as:

$$
\bar{H}_{P M, \rho}=\frac{4 B_{r} \delta_{P M}}{\mu_{P M} \pi \delta} \frac{\sin \left(\rho \Delta_{P M} / 2\right)}{\rho} e^{j \rho \vartheta_{r}},
$$

where $B_{r}$ is the remanence flux density, $\delta_{P M}$ the magnet thickness, $\mu_{P M}$ the magnet permeability, $\Delta_{P M}$ the permanent magnet span on the rotor surface, $\delta$ the magnetic air-gap and $\theta_{r}$ the rotor position in electrical radians. Considering the air magnetic permeability $\mu_{0}$, it is possible to get the flux density at the air-gap from (1) as:

$$
\bar{B}_{P M, \rho}=\mu_{0} \bar{H}_{P M, \rho},
$$

When the machine rotates with a generic speed, the flux generated by the PMs is linked to the stator windings. At no load the variation of the flux in the time generates the BEMF $e_{\rho}$. The BEMF amplitude can be expressed also as a function of the machine geometrical parameters as:

$$
\bar{e}_{\rho}=2 R l N \omega_{p} p B_{P M, \rho} k_{w, \rho},
$$

where $R$ is the stator inner radius, $l$ is the steak length, $N$ is the turns per phase number, $\omega_{p}$ is the mechanical pulsation, $p$ is the pole pairs number and $k_{w, \rho}$ is the winding factor.

The winding factor can be written as:

$$
k_{w, \rho}=\frac{\sum_{y=1}^{N c} \sin \left(\rho \frac{\Delta \psi_{y}}{2}\right) e^{j \rho \psi_{y}}}{p q}
$$

where $\psi_{y}$ is the angular shift from the magnetic axis of the $\mathrm{y}$-th coil and the magnetic axis of the relative phase and $\mathrm{Nc}$ is the coils number and $\mathrm{q}$ the number per pole per phase.

Fig. 1 shows the flux density at the air-gap with respect to the PM span on the rotor $\Delta_{P M}$ for the following spatial harmonics: $1^{\text {st }}, 3^{\text {rd }}, 5^{t h}, 7^{t h}, 9^{t h}, 11^{t h}, 13^{t h}$. The values used for the computation of the parameters are summarised in Table I. The BEMF evaluation is shown in Fig. 2. It is worth to notice that the BEMF has different signs, for some harmonics, with respect to the flux density at the air-gap and the same amplitudes. This is due to the winding factor that for this 3x3-phase machine arrangement alternates the values 1 and -1 for the different harmonics. From Fig. 2, it is clear that the BEMF ratio, between a generic spatial harmonic and the fundamental one, changes with the PM span, $\Delta_{P M}$.

\section{B. Equations for current harmonics injection}

It has been shown how the machine average torque can be increased by injecting current temporal harmonics higher than the fundamental, creating an interaction with the spatial harmonics of higher order, generated by rotor PMs [13]- [14]. The analytical calculation of the optimum third harmonic current amplitude to maximise the torque, keeping constant the RMS current, is derived as:

$$
\begin{gathered}
a_{3}=\frac{e_{3}}{e_{1}}, \\
I^{*}=\frac{1}{\sqrt{1+\left(\frac{e_{3}}{e_{1}}\right)^{2}}} I_{p},
\end{gathered}
$$

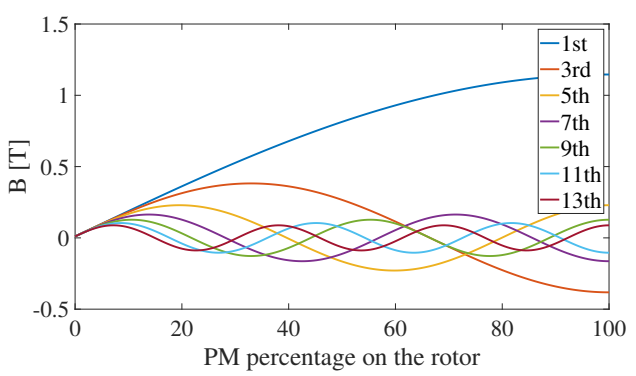

Fig. 1. Analytical flux density evaluation at the air-gap with respect to the magnets span on the rotor surface of a SPM machine.

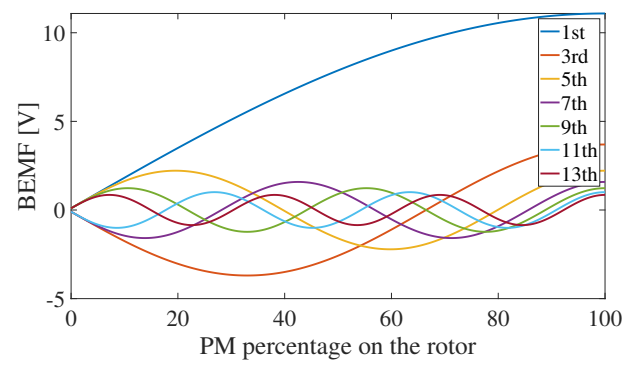

Fig. 2. Analytical BEMF evaluation with respect to the magnets span on the rotor surface of a SPM machine. 
TABLE I

MACHINE PARAMETERS

\begin{tabular}{ccc}
\hline Parameters & Values & Units \\
\hline$B_{r}$ & 1.2 & $\mathrm{~T}$ \\
$\delta_{P M}$ & 3 & $\mathrm{~mm}$ \\
$\mu_{0}$ & $4 \pi * 10^{-7}$ & $\mathrm{H} / \mathrm{m}$ \\
$\mu_{r}$ & 1 & $\mathrm{H} / \mathrm{m}$ \\
$\mu_{P M}$ & $4 \pi * 10^{-7}$ & $\mathrm{H} / \mathrm{m}$ \\
$\delta$ & 4 & $\mathrm{~mm}$ \\
$g$ & 1 & $\mathrm{~mm}$ \\
$\omega_{p}$ & 157.07 & $\mathrm{rad} / \mathrm{s}$ \\
$R$ & 70 & $\mathrm{~mm}$ \\
$l$ & 220 & $\mathrm{~mm}$ \\
$p$ & 2 & - \\
$N$ & 1 & - \\
\hline
\end{tabular}

where $a_{3}$ is the optimum third harmonic amplitude with respect to the fundamental, which is calculated as the ratio between the $3^{\text {rd }}$ and the $1^{\text {st }}$ BEMF harmonics. In eq. (6) the $I^{*}$ is the new phase current with injection and $I_{p}$ is the phase current without injection.

In this work, the analytical equation for the current harmonic injection, keeping the same RMS, is generalised for infinite harmonics. The optimum current amplitude, to inject for each harmonic, and the generalised phase current $\left(I^{*}\right)$ equation can be expressed as:

$$
\begin{gathered}
a_{i}=\frac{e_{i}}{e_{1}}, i=3,5,7, \ldots, \infty \\
I^{*}=\frac{I_{p}}{\sqrt{1+\sum_{i=3}^{\infty} a_{i}^{2}}},
\end{gathered}
$$

Therefore, the amplitude current of each injected harmonics is:

$$
\begin{aligned}
I_{1} & =I^{*} \\
I_{i}=I^{*} a_{i}, i & =3,5,7, \ldots, \infty
\end{aligned}
$$

If the torque is expressed as a function of the BEMF, it is possible to know its correlation with the $\Delta_{P M}$ variation. This is investigated for both cases under study, with and without current harmonics injection. The electromagnetic torque for a $3 \times 3$-phase system without current harmonics injection can be written as:

$$
T=\frac{9}{2} \frac{I_{p} e_{1}}{\omega_{p}},
$$

When the harmonics are injected in the machine, the torque can be calculate considering each contribution, independently:

$$
\begin{gathered}
T_{1}=\frac{9}{2} \frac{I_{1} e_{1}}{\omega_{p}} \\
T_{i}=\frac{9}{2} \frac{I_{i} e_{i}}{\omega_{p}}, i=3,5,7, \ldots, \infty
\end{gathered}
$$

By combining each torque contribution, the total torque can be defined as:

$$
T_{n e w}=T_{1}+\sum_{i=3}^{\infty} T_{i}
$$

TABLE II

TORQUE INCREMENT BY CURRENT INJECTION

\begin{tabular}{cc}
\hline$\Delta_{P M}$ & Torque increment \\
\hline $20 \%$ & $+52.7 \%$ \\
$40 \%$ & $+16.9 \%$ \\
$60 \%$ & $+4.9 \%$ \\
$80 \%$ & $+3.6 \%$ \\
$100 \%$ & $+9.4 \%$ \\
\hline
\end{tabular}

The temporal harmonics for the current injection technique are the ones of odd order $\mathrm{z}=2 \mathrm{k}+1$, where $\mathrm{k}=1,2$, $3, \ldots, \infty$. In this case, the maximum order considered is $13^{t h}$, neglecting the higher order harmonics. In Fig. 3 is shown the trend of torque as a function of $\Delta_{P M}$ for both fundamental only and with the injection of all the harmonics considered. The phase current amplitude is $I_{p}=683.7 \mathrm{~A}$. It is worth to notice that the torque increment, given by the injection of all the considered current harmonics, is higher for all PM span $\Delta_{P M}$. In Table II a summary of the torque increment with respect to fundamental only is reported. The different increments are given to the higher BEMF ratios for some values of $\Delta_{P M}$. Even in for some cases the potential torque increment is considerable, for example when $\Delta_{P M}$ is $20 \%$ and $40 \%$, the torque can be $+52.7 \%$ and $+16.9 \%$ higher, these are not considered to be a practical solution. However, for some rotor configurations, with a good PM coverage, it is possible to get a torque improvement. On the other hand, thanks to the harmonics injection technique, it is also possible to deliver a specific torque value, with a reduced amount of PMs. In order to have a complete understanding of the machine behaviour under the harmonics injection technique, for the same RMS current, it is worth to investigate its influence on both the peak phase current and voltage.

\section{Finite Element Validation}

Based on the findings shown in the previous section, the following analysis aims at demonstrating the benefits of the current injection with different harmonic components. In order to validate the analytical model, a FE analysis is carried out for two machines, namely M1 and M2, with the same geometry and two different permanent magnet span. The $\Delta_{P M}$ in M1

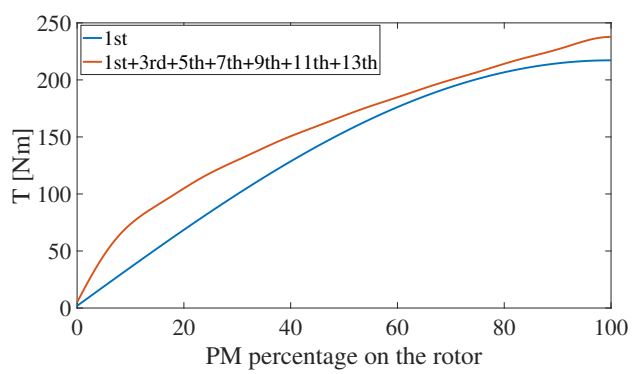

Fig. 3. Analytical torque evaluation with and without harmonics injection with respect to the magnets span on the surface rotor of a SPM machine. 
is $82 \%$ while in $\mathrm{M} 288 \%$, which corresponds to a reduction of PM volume of $6 \%$ in M1 with respect to M2. First M1 is analysed with and without harmonics injection to show the torque increment while keeping the same RMS current. Then, this is compared against the torque performance of M2, which has higher content of PM, supplying with only the fundamental current component.

The analyses are carried out considering linear materials. The proposed SPM machine geometry is shown in Fig. 4, highlighting the parameter $\Delta_{P M}$.

\section{A. Torque analysis}

Fig. 5 shows the torque comparison derived by means of FE analysis and with the analytical formulation. Fig. 5(a) presents the FE results only. For M1, it is possible to notice that with the harmonics injection the average torque is increased of $3.2 \%$ with respect to the case of fundamental supply only. This is still slightly higher, by $1 \%$, with respect to the torque delivered by M2 without harmonic injection, even if the permanent magnet content is reduced by $6 \%$. The same analysis has been carried out using the analytical model. The results are shown in Fig. 5(b). In this case, for M1, thanks to the currents injection there is torque increment by $3.7 \%$. In addition, the average torque is also higher for M1, when the considered harmonics are injected, compared to M2 without injection, in accordance to the trends resulting from the FE analysis (5(a)). The increment in this case is $1.4 \%$. It is worth to notice that the average torque values estimated analytically are slightly higher compared to the FE ones. This is due to the fact that the proposed analytical model does not take into the account the slotting effect and it assumes that the flux density at the air-gap is radial. Always from Fig. 5(a) it can be highlighted that the current harmonic injection is also slightly affecting the torque ripple. For M1, when the harmonics are injected the ripple is increasing from $10.8 \%$ to $12.2 \%$. However, with respect to machine $\mathrm{M} 2$ there is a reduction of $1.8 \%$, from $14 \%$ to $12.2 \%$. This is also showing that the combination of permanent magnet span and current harmonic injection can be refined in order to minimise the torque ripple in the machine. This is different with respect to other torque ripple minimisation techniques reported in literature where the injection of harmonics is not used to to increase the torque, but

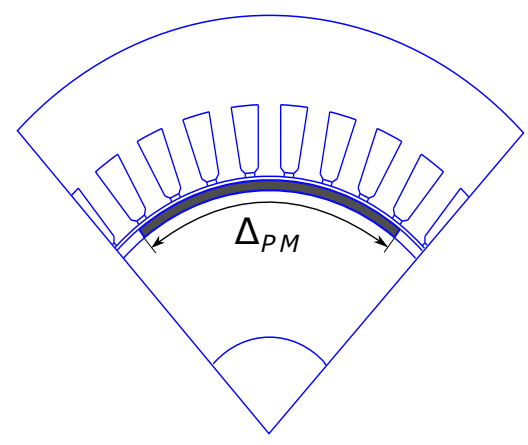

Fig. 4. Sketch of the SPM machine under analysis. to eliminate some harmonic components to reduce the torque ripple [19].

\section{B. Current and voltage validation}

From a physical point of view, it is not always obvious to understand how the injection of currents, with higher order harmonic with respect to the fundamental, can affect the peak currents and voltages. In this subsection these aspects for both machines configurations, M1 and M2. The expression of the currents fed to the machine windings, with and without current harmonics injection technique, can be represented as follows:

$$
\begin{gathered}
i(t)=I_{p} \sin (\omega t), \\
i(t)=\sum_{i=1}^{13} I_{i} \sin (i \omega t),
\end{gathered}
$$

with $i=1,3,5,7,9,11,13$.

The phase current calculated both analytically and by means of FE simulations is compared in Fig. 6. From the graph it is difficult to appreciate any difference. The RMS current values are the same with and without harmonic injection. More in detail, the FE analysis shows that the peak current, with harmonics injection is $16.3 \%$ lower with respect to the fundamental peak without current injection. The same waveform from the analytical model is plotted, highlighted in dashed line, showing a good match with the FE results. This means that even in case of harmonic injection, for the case under study, the inverter switches do not need to be oversized, thanks to the lower peak current.

The phase current with the harmonics injection is based on the BEMF ratios. The BEMF amplitudes comparison between
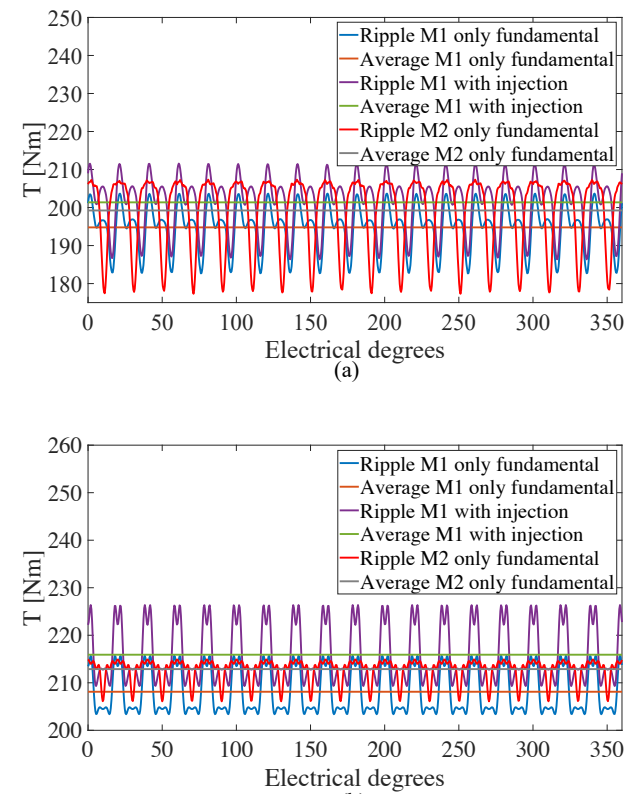

(b)

Fig. 5. (a) FE torque comparison between the machine 1 with and without harmonics injection and the machine 2 with only the fundamental.- (b) Analytical torque comparison between the machine 1 with and without harmonics injection and the machine 2 with only the fundamental. 
TABLE III

Phase RESistance AND INDUCTANCES VALUES For the PHASE X (A2)

\begin{tabular}{ccc}
\hline Parameters & Values & Units \\
\hline$r$ & $2.6410^{-4}$ & $\Omega$ \\
$L x$ & $9.1510^{-6}$ & $\mathrm{H}$ \\
$M x 1$ & $5.5210^{-6}$ & $\mathrm{H}$ \\
$M x 3$ & $5.5210^{-6}$ & $\mathrm{H}$ \\
$M x 4$ & $-7.8810^{-7}$ & $\mathrm{H}$ \\
$M x 5$ & $-2.3610^{-6}$ & $\mathrm{H}$ \\
$M x 6$ & $-3.9410^{-6}$ & $\mathrm{H}$ \\
$M x 7$ & $-3.9410^{-6}$ & $\mathrm{H}$ \\
$M x 8$ & $-2.3610^{-6}$ & $\mathrm{H}$ \\
$M x 9$ & $-7.8810^{-7}$ & $\mathrm{H}$ \\
\hline
\end{tabular}

analytical and FE for M1 is shown in Fig. 7. The analytical voltage for the phase $\mathrm{x}(\mathrm{A} 2)$ is calculated as:

$$
v_{x}=r i_{x}+L_{x} \frac{d i_{x}}{d t}+\sum_{y=1, y \neq x}^{9} M_{x y} \frac{d i_{y}}{d t}+e_{x},
$$

where $r$ is the phase resistance, $L_{x}$ is the auto-inductance for the phase x (A2), Mxy are the mutual-inductances for the phase $\mathrm{x}(\mathrm{A} 2)$ due to the other stator phases $\mathrm{y}$ and $e_{x}$ is the BEMF for the phase $\mathrm{x}$. The phase resistance and the inductances values, with $N=1$, for the phase x (A2) are shown in TABLE III. The phase voltage waveforms for both analytical model and FE are shown in Fig. 8(a) and Fig. 8(b). Both analyses show that the injection, for the proposed PM span $\Delta_{P M}$ configuration, does not increase the peak voltage significantly. Indeed, for the FE analysis, the peak voltage for M1 with the injection is higher by $8.3 \%$ compared to M1 and by $5.5 \%$ with respect to $\mathrm{M} 2$ with the control without injection. The analytical model results presents a peak voltage, for M1 with injection, higher by $6.8 \%$ with respect to both $\mathrm{M} 1$ and M2 without injection.

\section{Summary}

A summuary results for both analytical and FE analyses are shown in Table IV. It is possible to notice that there is a slight displacement between the analytical and FE torque analyses. This could be due to the fact that the magnetic airgap $\delta$ is enough large for the considered machine $(4 \mathrm{~mm})$.

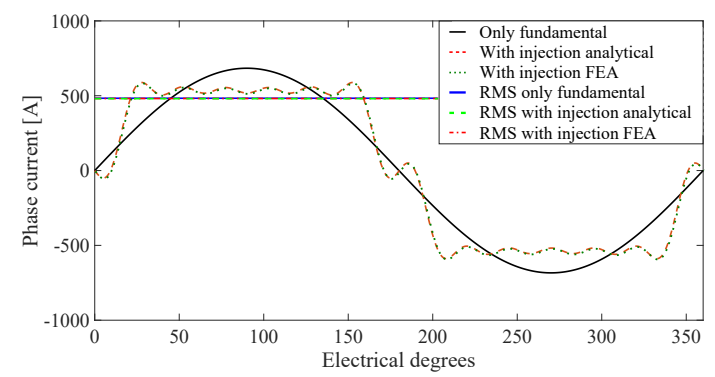

Fig. 6. Phase current comparison between the machine 1 with and without harmonics injection and the machine 2 with only the fundamental for both analytical and FEA.
TABLE IV

COMPARISON ANALYSIS

\begin{tabular}{ccc}
\hline- & M1 Analytical & M1 FE \\
\hline I peak no inj [A] & 683.7 & 683.7 \\
V peak no inj [V] & 11.8 & 13.8 \\
T no inj [Nm] & 208 & 195 \\
I peak with inj [A] & 588 & 582 \\
V peak with inj [V] & 12.6 & 14.8 \\
T with inj [Nm] & 216 & 201 \\
T inc. [\%] & 3.7 & 3.2 \\
\hline- & M2 Analytical & M2 FE \\
\hline I peak no inj [A] & 683.7 & 683.7 \\
V peak no inj [V] & 11.8 & 14.0 \\
T no inj [Nm] & 213 & 199 \\
I peak with inj [A] & - & - \\
V peak with inj [V] & - & - \\
T with inj [Nm] & - & - \\
T inc. [\%] & - & - \\
\hline
\end{tabular}

Therefore, the flux density at the air-gap is not total radial but it presents a not negligible tangential component, and the proposed analytical model cannot take into the account that. However, the simplified analytical model results can be considered acceptable for understanding the main phenomenon of the machine under harmonics injection and which PM span configuration could be more suitable for the proposed harmonics injection technique linked with a possible PM reduction.

\section{CONCLUSION}

This work investigates on the PM reduction, without affecting the torque performance and Joule losses, for SPM machines. First of all, an analytical model is carried out showing how the no-load flux density, for different spatial harmonics, changes with the $\Delta_{P M}$ variation. Then, the same analysis is carried out for the BEMF spatial harmonics studying a $3 \times 3$-phase winding arrangement. In addition, equations for the optimum harmonic injection amplitudes to maximise the torque, while keeping constant the RMS current with respect to the current control with the only fundamental component, are presented. The current amplitude of each injected harmonics

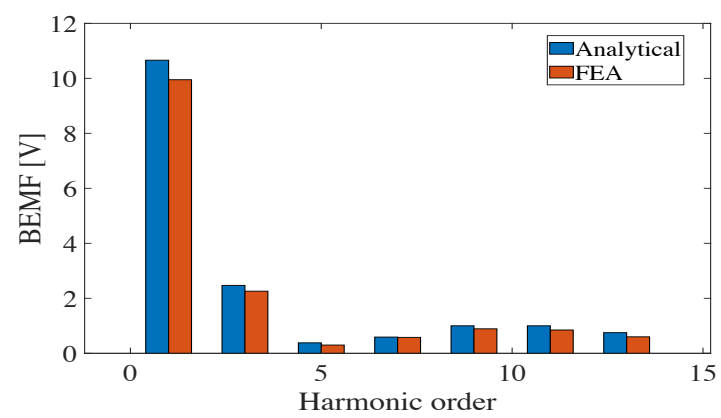

Fig. 7. BEMF comparison between analytical model and FE analysis for the machine with the $82 \%$ of PM span (M1). 

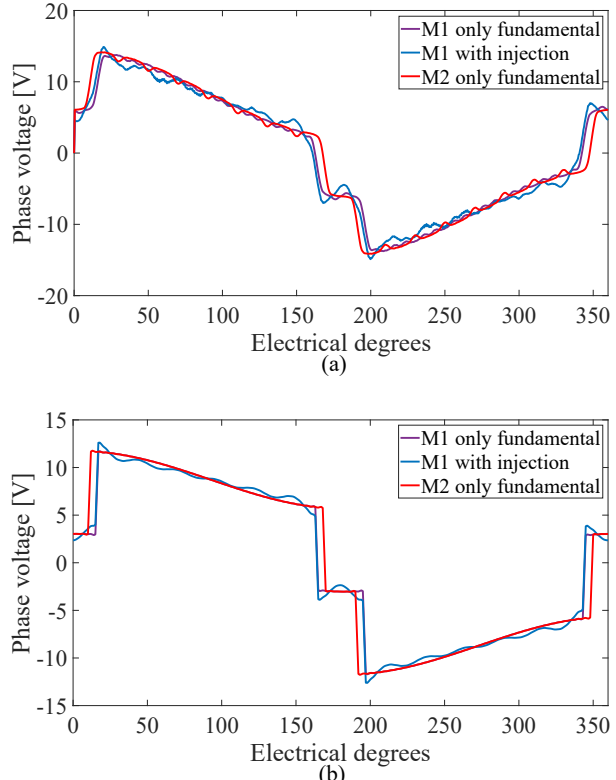

Fig. 8. (a) FE phase voltage comparison between the machine 1 with and without harmonics injection and the machine 2 with only the fundamental. (b) Analytical phase voltage comparison between the machine 1 with and without harmonics injection and the machine 2 with only the fundamental. For the analytical voltage calculation 1000 spatial harmonics are taken into the account

depends of the BEMF ratios. The proposed current harmonics injection technique takes into the account the following temporal harmonics: $3^{r d}, 5^{t h}, 7^{\text {th }}, 9^{\text {th }}, 11^{\text {th }}, 13^{\text {th }}$. The analytical torque comparison between the current control with and without harmonics injection is shown as a function of the $\Delta_{P M}$ variation, highlighting the torque increment once the harmonics injection is applied. The analytical model is validated via FEA which compares a SPM machine with $82 \%$ of PM span (M1), with and without harmonics injection, and a SPM machine with $88 \%$ of PM span (M2), feeding with only the fundamental current component. The analysis shows, for the case under study, that it is possible to reduce the PM content of the $6 \%$, once that the considered current harmonics are injected, without affecting torque performance, peak current and peak voltage. The slight displacement between analytical and FE analyses can be justified by the fact that the flux at the air-gap is not total radial for the FE solution. However, the simplified analytical approach can give important information on which PM percentage on the rotor surface is better than others for injecting high current temporal harmonic components in order to increase the torque or to reduce the PM content, keeping the same RMS current value of the current control without injection, thus the same Joule losses.

\section{REFERENCES}

[1] C. Gerada, M. Galea and A. Kladas, "Electrical machines for aerospace applications," 2015 IEEE Workshop on Electrical Machines Design, Control and Diagnosis (WEMDCD), Torino, 2015, pp. 79-84, doi: 10.1109/WEMDCD.2015.7194513.

[2] S. Zhang et al., "Permanent magnet technology for electric motors in automotive applications," 2012 2nd International Electric Drives
Production Conference (EDPC), Nuremberg, 2012, pp. 1-11, doi: 10.1109/EDPC.2012.6425118.

[3] H. Tahanian, M. Aliahmadi and J. Faiz, "Ferrite Permanent Magnets in Electrical Machines: Opportunities and Challenges of a Non-Rare-Earth Alternative," in IEEE Transactions on Magnetics, vol. 56, no. 3, pp. 1-20, March 2020, Art no. 900120, doi: 10.1109/TMAG.2019.2957468.

[4] I. Boldea, L. N. Tutelea, L. Parsa and D. Dorrell, "Automotive Electric Propulsion Systems With Reduced or No Permanent Magnets: An Overview," in IEEE Transactions on Industrial Electronics, vol. 61, no. 10, pp. 5696-5711, Oct. 2014, doi: 10.1109/TIE.2014.2301754.

[5] M. Barcaro and N. Bianchi, "Interior PM Machines Using Ferrite to Replace Rare-Earth Surface PM Machines," in IEEE Transactions on Industry Applications, vol. 50, no. 2, pp. 979-985, March-April 2014, doi: 10.1109/TIA.2013.2272549.

[6] S. S. R. Bonthu, A. Arafat and S. Choi, "Comparisons of Rare-Earth and Rare-Earth-Free External Rotor Permanent Magnet Assisted Synchronous Reluctance Motors," in IEEE Transactions on Industrial Electronics, vol 64, no. 12, pp. 9729-9738, Dec. 2017, doi: 10.1109/TIE.2017.2711580.

[7] Q. Chen, G. Liu, W. Zhao, M. Shao and Z. Liu, "Design and Analysis of the New High-Reliability Motors With Hybrid Permanent Magnet Material," in IEEE Transactions on Magnetics, vol. 50, no. 12, pp. 110, Dec. 2014, Art no. 8207010, doi: 10.1109/TMAG.2014.2342662.

[8] L. Parsa, "On advantages of multi-phase machines," 31st Annual Conference of IEEE Industrial Electronics Society, 2005. IECON 2005., Raleigh, NC, 2005, pp. 6 pp.-, doi: 10.1109/IECON.2005.1569139.

[9] A. S. Abdel-Khalik, A. M. Massoud and S. Ahmed, "Application of Standard Three-Phase Stator Frames in Prime Phase Order Multiphase Machine Construction," in IEEE Transactions on Industrial Electronics, vol. 66, no. 4, pp. 2506-2517, April 2019, doi: 10.1109/TIE.2018.2840497.

[10] M. Onsal, Y. Demir and M. Aydin, "A New Nine-Phase Permanent Magnet Synchronous Motor With Consequent Pole Rotor for High-Power Traction Applications," in IEEE Transactions on Magnetics, vol. 53, no. 11, pp. 1-6, Nov. 2017, Art no. 8700606, doi: 10.1109/TMAG.2017.2709788

[11] M. Barcaro, N. Bianchi and F. Magnussen, "Analysis and tests of a dual three-phase 12-slot 10-pole permanent magnet motor," 2009 IEEE Energy Conversion Congress and Exposition, San Jose, CA, 2009, pp. 3587-3594, doi: 10.1109/ECCE.2009.5316094.

[12] J. Karttunen, S. Kallio, P. Peltoniemi, P. Silventoinen and O. Pyrhönen, "Decoupled Vector Control Scheme for Dual Three-Phase Permanent Magnet Synchronous Machines," in IEEE Transactions on Industrial Electronics, vol. 61, no. 5, pp. 2185-2196, May 2014, doi: 10.1109/TIE.2013.2270219.

[13] K. Wang, Z. Q. Zhu, Y. Ren and G. Ombach, "Torque Improvement of Dual Three-Phase Permanent-Magnet Machine With Third-Harmonic Current Injection," in IEEE Transactions on Industrial Electronics, vol. 62, no. 11, pp. 6833-6844, Nov. 2015, doi: 10.1109/TIE.2015.2442519.

[14] Z. Y. Gu, K. Wang, Z. Q. Zhu, Z. Z. Wu, C. Liu and R. W. Cao, ’Torque Improvement in Five-Phase Unequal Tooth SPM Machine by Injecting Third Harmonic Current," in IEEE Transactions on Vehicular Technology, vol. 67, no. 1, pp. 206-215, Jan. 2018, doi: 10.1109/TVT.2017.2771752.

[15] Y. Hu, Z. Q. Zhu and M. Odavic, "Torque Capability Enhancement of Dual Three-Phase PMSM Drive With Fifth and Seventh Current Harmonics Injection," in IEEE Transactions on Industry Applications, vol. 53, no. 5, pp. 4526-4535, Sept.-Oct. 2017, doi: 10.1109/TIA.2017.2707330.

[16] M. Slunjski, M. Jones and E. Levi, "Analysis of a Symmetrical Nine-phase Machine with Highly Non-Sinusoidal Back-Electromotive Force," IECON 2018 - 44th Annual Conference of the IEEE Industrial Electronics Society, Washington, DC, 2018, pp. 6229-6234, doi: 10.1109/IECON.2018.8591091.

[17] A. S. Abdel-Khalik, S. Mostafa Gadoue, M. I. Masoud and B. W. Wiliams, "Optimum Flux Distribution With Harmonic Injection for a Multiphase Induction Machine Using Genetic Algorithms," in IEEE Transactions on Energy Conversion, vol. 26, no. 2, pp. 501-512, June 2011, doi: 10.1109/TEC.2010.2093139.

[18] M. Slunjski, O. Stiscia, M. Jones and E. Levi, "General Torque Enhancement Approach for a Nine-Phase Surface PMSM with Built-in Fault Tolerance," in IEEE Transactions on Industrial Electronics, doi: 10.1109/TIE.2020.3007053.

[19] G. Lee, S. Kim, J. Hong and J. Bahn, "Torque Ripple Reduction of Interior Permanent Magnet Synchronous Motor Using Harmonic Injected Current," in IEEE Transactions on Magnetics, vol. 44, no. 6, pp. 15821585, June 2008, doi: 10.1109/TMAG.2008.915776. 